\title{
POTENCIAL NUTRITIVO DE FRUTOS DE PITANGÃO (Eugenia neonitida, SOBRAL) ${ }^{1}$
}

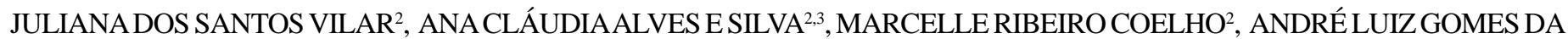 \\ SILVA $^{4}$, ARMANDO UBIRAJARADE OLIVEIRASABAA SRUR ${ }^{5}$
}

\begin{abstract}
RESUMO - Eugenia neonitida, Sobral (Myrtaceae) ocorre naturalmente nas restingas dos Estados do Rio de Janeiro e Espírito Santo, possui hábito arbustivo e pode alcançar até $2,5 \mathrm{~m}$ de altura. Floresce entre os meses de outubro e novembro, frutificando de novembro a janeiro. Seus frutos, popularmente chamados de "Pitangão", são oblongos, e durante o processo de amadurecimento sua cor evolui do verde até o amarelo vivo quando maduro. Apresentam sabor agridoce e exalam fragrância agradável, tornando-os amplamente apreciados pela população local. Determinações analíticas revelaram que $100 \mathrm{~g}$ de polpa deste fruto contêm alto teor de umidade (93,2\%) e baixo valor calórico, $54,21 \mathrm{kcal}$, oriundo de 3,21 $\pm 0,25 \mathrm{~g}$ de lipídeos, $2,2 \pm 0,86 \mathrm{~g}$ de proteínas, $0,55 \pm 0,03 \mathrm{~g}$ de carboidratos, além de $6 \mathrm{~g}$ de sólidos solúveis e alta concentração de minerais, com destaque para o sódio, $480,8 \mathrm{mg} .100 \mathrm{~g}^{-1}$ de polpa do fruto. A acidez total é de $1,38 \pm 0,03 \mathrm{~g} \mathrm{de} \mathrm{NaOH} .100 \mathrm{~g}^{-1}, \mathrm{o} \mathrm{pH}$ é 2,85 e a concentração de ácido ascórbico é de $17,86 \pm 0,06 \mathrm{mg} 100 \mathrm{~g}^{-1}$. O valor de $\beta$-caroteno é de $60 \pm 0,04 \mathrm{mg}$. $100 \mathrm{~g}^{-1}$. Comparando-se a polpa do pitangão com a da pitanga (Eugenia uniflora L.), foram constatados maiores valores nutricionais para a primeira. O pitangão pode ser uma ótima fonte de macro e micronutrientes para alimentação humana, embora ainda não seja cultivada nem comercializada.
\end{abstract}

Termos para indexação: Myrtaceae, Eugenia neonitida, pitangão, informações nutricionais.

\section{NUTRITIVE POTENTIAL OF PITANGÃO (Eugenia neonitida, SOBRAL) FRUITS AND SEEDS}

ABSTRACT - Eugenia neonitida, Sobral (Myrtaceae) occurs naturally in the states of Rio de Janeiro and Espírito Santo. Generally, it is a shrub, but it can reach up to 2,5m of height. It blooms from August to November and fructifies from October to December. Their fruits, popularly called "Pitangão", are oblong and during the maturation process the fruit color turns from green to bright yellow when ripe. They present a bittersweet taste and a pleasant fragrance, what makes much appreciated by local population. Analytical determinations revealed that $100 \mathrm{~g}$ of pulp possess high concentration of water and low caloric value, 54,21 kcal, originated from 3,21 $\pm 0,25 \mathrm{~g}$ of lipids, 2,2 $\pm 0,86 \mathrm{~g}$ of proteins, $0,55 \pm 0,03 \mathrm{~g}$ of carbohydrates, beyond $6 \mathrm{~g}$ of soluble solids and high concentration of minerals, specially sodium with $480,8 \mathrm{mg} .100 \mathrm{~g}^{-1}$. Total acidity is $1,38 \pm 0,03 \mathrm{~g}$ of $\mathrm{NaOH} .100 \mathrm{~g}^{-1}$, $\mathrm{pH}$ is 2,85 and concentration of ascorbic acid is $17,86 \pm 0,06 \mathrm{mg} .100 \mathrm{~g}^{-1}$. The value of $\beta$-carotene is $60 \pm 0,04 \mathrm{mg} .100 \mathrm{~g}^{-1}$. When compared the "Pitangão" pulp with of the Surinam cherry (Eugenia uniflora L.) it was verified larger nutritional values for the first one. The "Pitangão" can be a great source of macro and micronutrients for human nutrition, although its cultivation and commercialization is not implemented yet.

Index terms: Myrtaceae, Eugenia neonitida, pitangão, nutrition facts.

O Brasil, em função da enorme biodiversidade e condições edafoclimáticas, é um país com imenso potencial para obtenção de recursos vegetais naturais. A flora brasileira é rica em espécies frutíferas, destacando-se várias espécies de Myrtaceae, como a pitanga (Eugenia uniflora L.), o baguaçu (E. umbelliflora Berg), a cagaita (E. dysenterica DC.), entre outras.

Eugenia neonitida, Sobral (Myrtaceae) ocorre nas restingas dos Estados do Rio de Janeiro e Espírito Santo (Araújo \& Henriques, 1984), e seus frutos, popularmente chamados de "Pitangão", são amplamente apreciados pela população local.

Com o objetivo avaliar o potencial nutritivo da polpa e das sementes de frutos maduros de E. neonitida, Sobral coletados em seu habitat natural, foi realizada a coleta desses frutos quando estes apresentavam coloração amarela, em cinco indivíduos, em condições naturais na restinga de Grumari, localizada na região oeste do Município do Rio de Janeiro. Eugenia neonitida ocorre principalmente nas formações de pós-praia e arbustiva-aberta desta restinga (Argôlo, 2001). De cada indivíduo foi coletado um número máximo de 20 frutos.

A polpa e as sementes de frutos maduros foram removidas manualmente com auxílio de estiletes de aço inoxidável e acondicionadas separadamente em sacos de polietileno de cinco camadas com alta impermeabilidade e imediatamente congeladas até a realização das determinações.

A umidade foi mensurada pela remoção da água livre de amostras homogêneas de polpa e de sementes com auxílio de estufa a vácuo a $60^{\circ} \mathrm{C}$, conforme metodologia preconizada pelo Instituto Adolfo Lutz (1985).

O conteúdo de minerais totais (cinzas) foi determinado após ignição de toda a matéria orgânica das amostras de polpa e de sementes em mufla a $550^{\circ} \mathrm{C}$. As amostras foram previamente carbonizadas em chapas aquecidas e posteriormente submetidas à incineração (IAL, 1985).

Os macros e microminerais, tanto na polpa como nas sementes, foram determinados por espectrofotometria de massa com plasma indutivamente acoplado no modo semiquantitativo, utilizando o equipamento ELAN 6000 da Perkin Elmer-Sciex (AOAC, 1990).

Os teores de nitrogênio total foram determinados através do método micro-Kjeldhahl (AOAC, 1990). O conteúdo de nitrogênio encontrado em cada amostra foi multiplicado por 6,25 para se encontrar o percentual de proteína bruta (AACC, 1995).

A fração de lipídeos das sementes foi mensurada pelo método de Soxhlet utilizando-se como extrator o éter etílico, enquanto na polpa este teor foi determinado pelo procedimento preconizado por Bligh \& Dyer (1959), com a utilização de três solventes, clorofórmio, metanol e água, na proporção 2:1:0,5, respectivamente.

Os teores de sólidos solúveis foram determinados em amostra de polpa previamente filtrada para reduzir a interferência dos sólidos insolúveis. A leitura foi realizada a $20^{\circ} \mathrm{C}$, e os resultados, expressos em graus brix ( $\left.{ }^{\circ} \mathrm{Brix}\right)$ (IAL, 1985). O pH foi determinado em suspensões preparadas a partir da polpa e da semente (IAL, 1985).

\footnotetext{
${ }^{1}$ (Trabalho 38-2006). Recebido: 03-04-2006. Aceito para publicação: 25-08-2006.

2 Alunas do Curso de Graduação em Nutrição da Universidade Federal do Rio de Janeiro (UFRJ) e estagiárias do Departamento de Nutrição Básica e Experimental (DNBE) do Instituto de Nutrição Josué de Castro. Av. Pau Brasil, 211 Bloco J - $2^{\circ}$ andar, Cidade Universitária Rio de Janeiro - RJ - Brasil. CEP: 21941-900. Juliana dos Santos Vilar: jujuvilar@yahoo.com.br; Ana Cláudia Alves e Silva: anaclalves@bol.com.br; Marcelle Ribeiro Coelho: cellecoelho@hotmail.com

${ }^{3}$ Bolsista Apoio UFRJ

${ }^{4}$ Aluno de doutorado do Programa de Pós-graduação em Ciências Biológicas, Botânica da UFRJ.Quinta da Boa Vista - São Cristóvão - Rio de Janeiro - RJ -

Brasil. CEP: 20940-040. andrebotanico@gmail.com

${ }^{5}$ Professor orientador do DNBE - UFRJ. sabaasrur@yahoo.com.br
} 
O teor de ácidos orgânicos presentes na polpa foi determinado por titulação com $\mathrm{NaOH}$ a $0,1 \mathrm{M}$ tendo-se fenolftaleina como indicador (IAL, 1985), enquanto a concentração de ácido ascórbico foi determinada pelo método de Tillmans, usando-se como padrão solução de ácido ascórbico a $1 \%$ (IAL, 1985) e o teor de $\beta$ caroteno por espectrofotometria a $450 \mathrm{~nm}$, usando-se diclorometano redestilado como branco (IAL, 1985).

As análises foram realizadas em triplicata, e os cálculos de média e desvio-padrão foram realizados a partir do software Microsoft Excel, 2000

Eugenia neonitida possui hábito arbustivo, podendo alcançar até $2,5 \mathrm{~m}$ de altura e floresce entre os meses de outubro e novembro, frutificando de novembro a janeiro. Seus frutos são oblongos, em média com $3,18 \pm 0,7 \mathrm{~cm}$ de comprimento $(\mathrm{N}=15), 2,47$ $\pm 0,28 \mathrm{~cm}$ de largura $(\mathrm{N}=15)$ e pesam 7,31 $\pm 3,66 \mathrm{~g}(\mathrm{~N}=58)$. Durante o processo de amadurecimento, a cor dos frutos passa do verde até o amarelo vivo, quando maduro. As sementes apresentaram 1,39 \pm $0,22 \mathrm{~cm}$ de comprimento $(\mathrm{N}=19), 0,94 \pm 0,16 \mathrm{~cm}$ de largura $(\mathrm{N}=19) \mathrm{e}$ $1,2 \pm 0,52 \mathrm{~g}(\mathrm{~N}=69)$. A polpa do fruto apresenta sabor agridoce e exala uma fragrância concentrada. Muitos compostos voláteis são responsáveis pelo aroma dos frutos, sendo os terpênicos predominantes em frutos tropicais (Franco \& Shibamoto, 2000). Em Eugenia stipitata (Araçá-boi), Franco \& Shibamoto (2000) afirmaram que os compostos voláteis responsáveis pelo aroma desse fruto são caracterizados por um complexo padrão de sesquiterpenos.

A polpa deste fruto apresentou alta taxa de umidade, 93,2\% (Tabela 1), característica comum de frutos tropicais, como a acerola (Malpighia sp.) (Nogueira et al., 1996), a banana (Musa sp.) e a laranja (Citrus sp.) (Vasconcellos et al., 1999). Os teores de minerais totais da polpa e das sementes de pitangão são apresentados na Tabela 1. A polpa apresentou a maior concentração do que a semente para todos os minerais analisados. O sódio foi o de maior quantidade, 480,8mg.100 $\mathrm{g}^{-1}$. Nas sementes, a maior predominância foi de magnésio, 97,4mg.100g ${ }^{-1}$. Outras espécies de Myrtaceae, como Psidium guajava L. (goiaba) (Haag et al., 1993) e Eugenia uniflora L. (Philippi, 2002), também apresentam elevada concentração de minerais.

Comparando os dados deste trabalho com os de Philippi (2002), sobre as propriedades nutricionais de pitanga (E. uniflora), a qual possui grande potencial econômico (Bezerra et al., 1995; 1997) e que pertence ao mesmo grupo taxonômico e coabita a mesma região do pitangão ( $E$. neonitida), constataram-se, nesta última, proporções maiores de alguns nutrientes em relação a $E$. uniflora (Tabela 2), como a quantidade de proteínas, de lipídios e de minerais. Somente o teor de carboidratos totais é maior na pitanga do que no pitangão.

O teor médio de ácido ascórbico presente na polpa de Eugenia neonitida é de $17,86 \pm 0,06 \mathrm{mg} 100 \mathrm{~g}^{-1}$, valor considerado baixo, mas que pode ser comparado às concentrações de ácido ascórbico em outras frutas, como a pêra, a lima e a manga (Andrade et al., 2002). Esse valor atende em torno de $20 \%$ das necessidades deste nutriente para um indivíduo adulto saudável do sexo masculino e $24 \%$ das necessidades deste nutriente para um indivíduo adulto saudável do sexo feminino (IOM, 2000). A concentração de $\beta$ caroteno encontrada foi de $60 \pm 0,04 \mathrm{mg} \cdot 100 \mathrm{~g}^{-1}$. Segundo Philippi (2002), a conversão de carotenos em retinol ocorre a nível de intestino delgado e é influenciada pela ingestão de proteínas e lipídeos. Visto que o pitangão apresenta teores elevados de proteínas e lipídeos, quando comparados com a pitanga, este fruto possivelmente promove maior biodisponibilidade deste carotenóide.

Os frutos de Eugenia neonitida, em virtude de suas propriedades nutricionais e pelo seu sabor agridoce, possuem alto potencial para utilização tanto a nível doméstico quanto industrial, sendo assim sugerido o seu cultivo e comercialização.

TABELA 1 - Composição química da polpa dos frutos e sementes de Eugenia neonitida, Sobral.

\begin{tabular}{|c|c|c|}
\hline Parâmetros & Polpa & Semente \\
\hline Umidade (\%) & $93,2 \pm 0,02$ & $51,1 \pm 0,15$ \\
\hline Cinzas $(\%)$ & $0,32 \pm 0,06$ & $2,3 \pm 0,18$ \\
\hline Acidez (g de $\mathrm{NaOH} .100 \mathrm{~g}^{-1}$ ) & $1,38 \pm 0,03$ & - \\
\hline Proteínas (g. $\left.100 \mathrm{~g}^{-1}\right)$ & $2,2 \pm 0,86$ & $5,03 \pm 0,71$ \\
\hline Lipídeos $\left(\mathrm{g} .100 \mathrm{~g}^{-1}\right)$ & $3,21 \pm 0,25$ & $11,55 \pm 0,14$ \\
\hline Carboidratos To tais $\left(\mathrm{g} .100 \mathrm{~g}^{-1}\right)$ & $0,55 \pm 0,03$ & $28,94 \pm 1,86$ \\
\hline Sódio $\left(\mathrm{mg} .100 \mathrm{~g}^{-1}\right)$ & $480,8 \pm 0,66$ & $80 \pm 0,21$ \\
\hline Magnésio (mg.100g $\left.{ }^{-1}\right)$ & $145,2 \pm 0,65$ & $97,4 \pm 0,45$ \\
\hline Cálcio $\left(\mathrm{mg} .100 \mathrm{~g}^{-1}\right)$ & $81,3 \pm 0,35$ & $49 \pm 0,25$ \\
\hline Ferro $\left(\mathrm{mg}^{1} 100 \mathrm{~g}^{-1}\right)$ & $4,3 \pm 0,25$ & $2,5 \pm 0,35$ \\
\hline Zinco $\left(\mathrm{mg} .100 \mathrm{~g}^{-1}\right)$ & $4,5 \pm 0,25$ & $2,9 \pm 0,15$ \\
\hline Selênio $\left(\mathrm{mg} 100 \mathrm{~g}^{-1}\right)$ & $0,5 \pm 0,15$ & $0,5 \pm 0,06$ \\
\hline
\end{tabular}

TABELA 2 - Comparação entre alguns dos nutrientes presentes na polpa dos frutos de Eugenia neonitida, Sobral (pitangão) e de E. uniflora L. (pitanga) (Philippi, 2002).

\begin{tabular}{ccc}
\hline Nutrientes & Pitangão & Pitanga \\
\hline Proteínas $(\mathrm{g})$ & 2,2 & 0,8 \\
Lipídeos $(\mathrm{g})$ & 3,21 & 0,4 \\
Carboidratos Totais $(\mathrm{g})$ & 0,55 & 7,5 \\
Sódio $(\mathrm{mg})$ & 480,8 & 3 \\
Magnésio $(\mathrm{mg})$ & 145,2 & 12 \\
Cálcio (mg) & 81,3 & 9 \\
Ferro (mg) & 4,3 & 0,2 \\
\hline
\end{tabular}

\section{REFERÊNCIAS}

AACC - AMERICAN ASSOCIATION OF CEREAL CHEMISTS. Approved methods of the AACC. $9^{\text {th }}$ ed. Washington, D.C., 1995. v.2, p.119-120.

ANDRADE, R.S.M.; DINIZ, M.C.T.; NEVES, E.A.; NÓBREGA, J.A. Determinação e distribuição de ácido ascórbico em três frutos tropicais. Eclética Química, São Paulo, v.27, n. especial, p.1-8, 2002.

AOAC - ASSOCIATION OF OFFICIALANALYTICALCHEMISTS. Offcial methods of analysis. $9^{\text {th }}$ ed. Washington, D.C., 1990. $1298 \mathrm{p}$. 
ARAÚJO, D.S.D.; HENRIQUES, R.P.B. Análise florística das restingas do Estado do Rio de Janeiro. In: LACERDA, L.D.de; ARAÚJO, D.S.D.de; CERQUEIRA, R.; TURCQ, B. (Org.). Restingas: origem, estrutura, processos. Niterói: CEUFF, 1984. p.159-193.

ARGÔLO, A.M. Levantamento, caracterização fisionômica e comparação da restinga de Grumari - RJ, com outras restingas do Estado do Rio de Janeiro. 2001. 62f. Dissertação (Mestrado em Ciências Biológicas, Botânica) - Programa de Pós-Graduação em Ciências Biológicas - Botânica do Museu Nacional da Universidade Federal do Rio de Janeiro, Rio de Janeiro, 2001.

BEZERRA, J.E.F.; LEDERMAN, I.E.; PEDROSA, A.C.; DANTAS, A.P.; FREITAS, E.V. Perfomance of surinam cherry, Eugenia uniflora L. in Pernambuco, Brazil. II - Productive period 19891995. Acta Horticulturae, International Symposium Myrtaceae, Leuven, p.137-142, 1995.

BEZERRA, J.E.F., PREITAS, E.V., PEDROSA, A.C., LEDERMAN, I.E. \& DANTAS, A.P. Performance of Surinam Cherry (Eugenia uniflora L.) in Pernambuco, Brazil. II - Productive period 19891995. Acta Horticulturae, Leuven, v.452, p.137-142, 1997.

BLIGH, E.G.; DYER, W.J. A rapid method of total lipid extraction and purification. Canadian Journal of Biochemistry and Physiology, Ottawa, v. 37, n.8, p.911-917, 1959.

FRANCO, M.R.B.; SHIBAMOTO, T. Volatile composition of some brazilian fruits: umbu-caja (Spondias citherea), camu-camu (Myrciaria dubia), araçá-boi (Eugenia stipitata) and cupuaçu (Theobroma grandiflorum). Journal of Agricultural and Food Chemistry, Easton, v.48, p.1263-1265, 2000.
HAAG, H.P.; MONTEIRO, F.A.; WAKAKUPI, P.Y. Frutos de goiaba (Psidium guajava L.): desenvolvimento e extração de nutrientes. Scientia Agricola, Piracicaba, v.50, n.3, p.413-418, 1993.

INSTITUTO ADOLFO LUTZ. Normas analíticas do Instituto Adolfo Lutz: métodos químicos e físicos para análise de alimentos. 3.ed., São Paulo, 1985. v.1, 533p.

IOM - INSTITUTE OF MEDICINE, FOOD AND NUTRITION BOARD. Dietary reference intakes for vitamin $C$, vitamin $E$, selenium, and carotenoids. Washington, DC: National Academy Press, 2000. 509p.

NOGUEIRA, C.M.D.; MAIA, G.A.; OLIVEIRA, G.S.F.; FIGUEIREDO, R.W.; ORIÁ, H.F. Características físicas, físico-químicas e químicas de acerolas do clone UFC. Ciência Agronômica, Fortaleza, v.27, n.1/2, p.76-80, 1996.

PHILIPPI, S.T. Tabela de composição de alimentos: suporte para decisão nutricional. São Paulo: Coronraio, 2002.

VASCONCELLOS, L.M.H.; LAURIA, D.C.; SILVA, L.H.C.;TADDEI, J.F. Relação entre as massas úmida, seca e de cinza em materiais biológicos: uma ferramenta para amostragens em campo e análise de amostras. Química Nova, São Paulo, v.22, n.6, p.899893,1999 